\title{
Depth-based hand pose estimation: methods, data, and challenges
}

\author{
James Steven Supančič III · Gregory Rogez • Yi Yang • Jamie Shotton • \\ Deva Ramanan
}

Received: date / Accepted: date

\begin{abstract}
Hand pose estimation has matured rapidly in recent years. The introduction of commodity depth sensors and a multitude of practical applications have spurred new advances. We provide an extensive analysis of the state-of-the-art, focusing on hand pose estimation from a single depth frame. To do so, we have implemented a considerable number of systems, and will release all software and evaluation code. We summarize important conclusions here: (1) Pose estimation appears roughly solved for scenes with isolated hands. However, methods still struggle to analyze cluttered scenes where hands may be interacting with nearby objects and surfaces. To spur further progress we introduce a challenging new dataset with diverse, cluttered scenes. (2) Many methods evaluate themselves with disparate criteria, making comparisons difficult. We define a consistent evaluation criteria, rigorously motivated by human experiments. (3) We introduce a simple nearestneighbor baseline that outperforms most existing systems. This implies that most systems do not generalize beyond their training sets. This also reinforces the under-appreciated point that training data is as important as the model itself. We conclude with directions for future progress.
\end{abstract}

Keywords hand pose, RGB-D sensor, datasets, benchmarking

James Steven Supančič III, Gregory Rogez, Deva Ramanan University of California, Irvine

E-mail: jsupanci@uci.edu

Yi Yang

Baidu Institute of Deep Learning

Jamie Shotton

Microsoft Research

\section{Introduction}

Human hand pose estimation empowers many practical applications, for example sign language recognition [20, visual interfaces [23, and driver analysis [27. Recently introduced consumer depth cameras have spurred a flurry of new advances $[33,23,20,51,45,6,21,47,42,34$.

Motivation: Recent methods have demonstrated impressive results. But differing (often in-house) testsets, varying performance criteria, and annotation errors impede reliable comparisons 26. Indeed, a recent metalevel analysis of object tracking papers reveals that it is difficult to trust the "best" reported method in any one paper 29. In the field of object recognition, comprehensive benchmark evaluation has been vital for progress [13,11,8]. Our goal is to similarly diagnose the state-of-affairs, and to suggest future strategic directions, for depth-based hand pose estimation.

Contributions: Foremost, we contribute the most extensive evaluation of depth-based hand pose estimators to date. We evaluate 13 state-of-the-art hand-pose estimation systems across 4 testsets under uniform scoring criteria. Additionally, we provide a broad survey of contemporary approaches, introduce a new testset that addresses prior limitations, and propose a new baseline for pose estimation based on nearest-neighbor (NN) exemplar volumes. Surprisingly, we find that NN exceeds the accuracy of most existing systems. We organize our discussion along three axes: test data (Sec. 2), training data (Sec. 3), and model architectures (Sec. 4). We survey and taxonomize approaches for each dimension, and also contribute novelty to each dimension (e.g. new data and models). After explicitly describing our ex- 


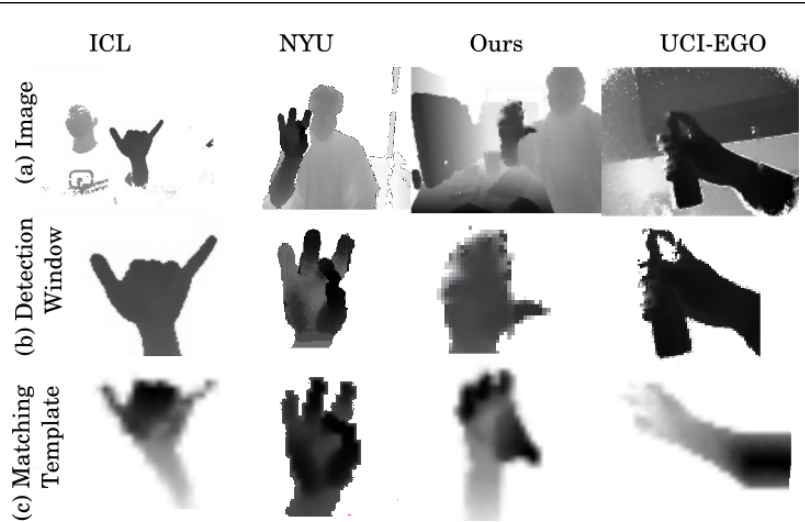

Fig. 1 NN Memorization: We evaluate a broad collection of hand pose estimation algorithms on different training and testsets under consistent evaluation criteria. Test sets which contained limited variety, in pose and range, or which lacked complex backgrounds were notably easier. To aid our analysis, we introduce a simple 3D exemplar (nearest-neighbor) baseline that both detects and estimates pose suprisingly well, outperforming most existing systems. We show the bestmatching detection window in (b) and the best-matching exemplar in (c). We use our baseline to rank dataset difficulty, compare algorithms, and illustrate the importance of training set design. We provide a detailed analysis of which problem types are currently solved, what open research challenges remain, and provide suggestions for future model architectures.

perimental protocol (Sec. 5), we end with an extensive empirical analysis (Sec. 6).

Preview: We foreshadow our conclusions here. When hands are easily segmented or detected, current systems perform quite well. However, hand "activities" involving interactions with objects/surfaces are still challenging (motivating the introduction of our new dataset). Moreover, in such cases even humans perform imperfectly. For reasonable error measures, annotators disagree $20 \%$ of the time (due to self and inter-object occlusions and low resolution). This has immediate implications for test benchmarks, but also imposes a challenge when collecting and annotating training data. Finally, our NN baseline illustrates some surprising points. Simple memorization of training data performs quite well, outperforming most existing systems. Variations in the training data often dwarf variations in the model architectures themselves (e.g., decision forests versus deep neural nets). Thus, our analysis offers the salient conclusion that "it's all about the (training) data".

Prior work: Our work follows in the rich tradition of benchmarking [11,37,9] and taxiomatic analysis [38, [10. In particular, Erol et al. [10] provided a review of hand pose analysis in 2007. Contemporary approaches have considerably evolved, prompted by the introduction of commodity depth cameras. We believe the time

\begin{tabular}{|c|c|c|c|c|c|c|c|}
\hline Dataset & Chal. & Scn. & Annot. & Frms. & Sub. & Cam. & Dist. (mm) \\
\hline ASTAR 51] & A & 1 & 435 & 435 & 10 & ToF & $270-580$ \\
Dexter 1 [42] & A & 1 & 3,157 & 3,157 & 1 & Both & $100-989$ \\
MSRA [33] & A & 1 & 2,400 & 2,400 & 6 & ToF & $339-422$ \\
ICL [45] & A & 1 & 1,599 & 1,599 & 1 & Struct & $200-380$ \\
\hline FORTH 28] & AV & 1 & 0 & 7,148 & 5 & Struct & $200-1110$ \\
NYU 47] & AV & 1 & 8,252 & 8,252 & 2 & Struct & $510-1070$ \\
\hline KTH 30] & AVC & 1 & 0 & 46,000 & 9 & Struct & NA \\
UCI-EGO 35] & AVC & 4 & 364 & 3,640 & 2 & ToF & $200-390$ \\
Ours & AVC & $10+$ & 23,640 & 23,640 & 10 & Both & $200-1950$ \\
\hline Challenges & (Chal.): & A-Articulation - \\
Viewpoint & C-Clutter
\end{tabular}

Table 1 Testing data sets: We group existing benchmark testsets into 3 groups based on the overall challenges addressed - articulation, viewpoint, and/or background clutter. We also tabulate the number of captured scenes, number of annotated versus total frames, number of subjects, camera type (structured light vs time-of-flight), and distance of the hand to camera. We introduce a new dataset (Ours) that contains a significantly larger range of hand depths (up to $2 \mathrm{~m})$, more scenes $(10+)$, more annotated frames $(24 \mathrm{~K})$, and more subjects (10) than prior work.

is right for another look. We do extensive cross-dataset analysis (by training and testing systems on different datasets [48]). Human-level studies in benchmark evaluation [22] inspired our analysis of human-performance. Finally, our NN-baseline is closely inspired by nonparametric approaches to pose estimation [39. In particular, we make use of volumetric depth features in a 3D scanning-window (or volume) framework, similar to 41. However, our baseline does not require SVM training or multi-cue features, making it considerably simpler to implement.

\section{Testing Data}

Test scenarios for depth-based hand-pose estimation have evolved rapidly. Early work evaluated on synthetic data, while contemporary work almost exclusively evaluates on real data. However, because of difficulties in manual annotation (a point that we will revisit), evaluation was not always quantitative - instead, it has been common to show select frames to give a qualitative sense of performance [1,7, 28, 30. However, we fundamentally assume that quantitative evaluation on real data will be vital for continued progress.

Test set properties: We have tabulated a list of contemporary test benchmarks in Table 1, giving URLs on our websit£. We refer the reader to the caption for a detailed summary of specific dataset properties. Per dataset, Fig. 2 visualizes the pose-space covered using multi-dimensional scaling (MDS). We plot both joint positions (in a normalized coordinate frame that is centered and scaled) and joint angles. Importantly,

\footnotetext{
1 http://www.ics.uci.edu/ jsupanci/\#HandData
} 
the position plot takes the global orientation (or camera viewpoint) of the hand into account while the angle plot does not. Most datasets are diverse in terms of joint angles but many are limited in terms of positions (implying they are limited in viewpoint). Indeed, we found that previous datasets make various assumptions about articulation, viewpoint, and perhaps most importantly, background clutter. Such assumptions are useful because they allow researchers to focus on particular aspects of the problem. However it is crucial to make such assumptions explicit [48, which much prior work does not. We do so below.

Articulation: Many datasets focus on pose estimation with the assumption that detection and overall hand viewpoint is either given or limited in variation. Example datasets include MSRA [33, A-Star [51, and Dexter [42. We focus on ICL [45] as a representative example for experimental evaluation because it has been used in multiple prior published works [45, 6].

Art. and viewpoint: Other testsets have focused on both viewpoint variation and articulation. FORTH 28] provides five test sequences with varied articulations and viewpoints, but these are unfortunately unannotated. In our experiments, we analyze the NYU dataset [47. because of its wider pose variation (see Fig. 2) and accurate annotations (see Sec. 3).

Art. + View. + Clutter: The most difficult datasets contain cluttered backgrounds that are not easy to segment away. These datasets tend to focus on "in-thewild" hands undergoing activities and interacting with nearby objects and surfaces. The KTH Dataset 30. provides a rich set of 3rd person videos showing humans interacting with objects. Unfortunately, annotations are not provided for the hands (only the objects). The UCI-EGO 35] dataset provides challenging sequences from an egocentric perspective, and so is included in our benchmark analysis.

Our testset: Our empirical evaluation will show that in-the-wild hand activity is still challenging. To push research in this direction, we have collected and annotated our own testset of real images (labeled as Ours in Table 1). As far as we are aware, our dataset is the first to focus on hand pose estimation across multiple subjects and multiple cluttered scenes. This is important, because any practical application must handle diverse subjects, scenes, and clutter.

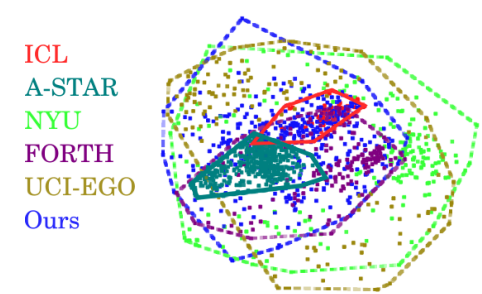

(a) Position

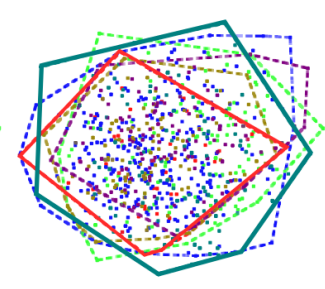

(b) Angle
Fig. 2 Pose variation: We use MDS (multi-dimensional scaling) to plot the pose space covered by various hand datasets. For each testset, we plot the convex hull of its poses. We plot joint positions (left) and joint angles (right). In terms of joint angle coverage (which does not consider the "root" orientation of the hand itself), most datasets are similar. In terms of joint position, some datasets are limited because they consider a smaller range of viewpoints (e.g., ICL and A-STAR). We further analyze various assumptions made by datasets in the text.

\section{Training Data}

Here we discuss various approaches for generating training data. Real annotated training data has long been the gold standard for supervised learning. However, the generally accepted wisdom (for hand pose estimation) is that the space of poses is too large to manually annotate. This motivates approaches to leverage synthetically generated training data, discussed further below.

Real data + manual annotation: Arguably, the space of hand poses exceeds what can be sampled with real data. Our experiments identify a second problem: perhaps surprisingly, human annotators often disagree on pose annotations. For example, in our testset, human annotators visually disagreed on $20 \%$ of pose annotations (given a visually-acceptable threshold of 20mm) as plotted in Fig. 14. These disagreements arise from limitations in the raw sensor data, either due to poor resolution or occlusions (as shown in Sec. 5.2). These ambiguities are often mitigated by placing the hand close to the camera [45,33,51. As an illustrative example, we evaluate the ICL training set [45].

Real data + automatic annotation: Data gloves directly obtain automatic pose annotations for real data [51]. However, they require painstaking per-user calibration and distort the hand shape that is observed in the depth map. Alternatively, one could use a "passive" motion capture system. We evaluate the NYU training set 47. that annotates real data by fitting (offline) a skinned 3D hand model to high-quality 3D measurements.

Quasi-synthetic data: Augmenting real data with geometric computer graphics models provides an attractive 


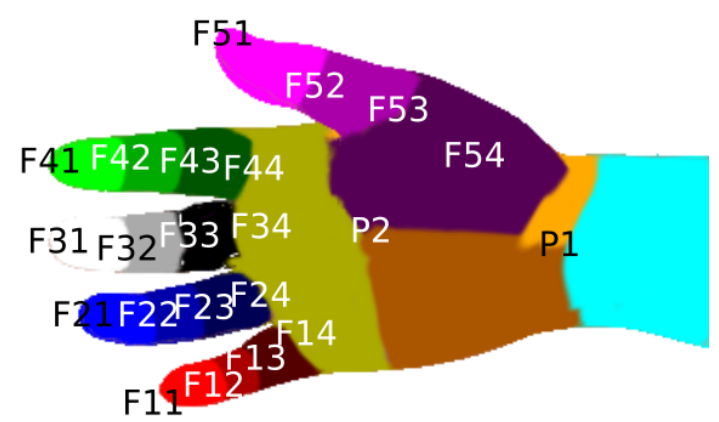

Fig. 3 libhand joints: We use the above joint identifiers to describe how we sample poses (for libhand) in table 2 Please see http://www.libhand.org/ for more details on the joints and their parameters.

solution. For example, one can apply geometric transformations (e.g., rotations) to both real data and its annotations 45]. If multiple depth cameras are used to collect real data (that is then registered to a model), one can synthesize a larger set of varied viewpoints [47]. Finally, mimicking the noise and artifacts of real data is often important when using synthetic data. Domain transfer methods [6] learn the relationships between a small real dataset and large synthetic one.

Synthetic data: Another hope is to use data rendered by a computer graphics system. Graphical synthesis sidesteps the annotation problem completely: precise annotations can be rendered along with the features. When synthesizing novel exemplars, it is important define a good sampling distribution. The UCI-EGO training set 35. synthesizes data with an egocentric prior over viewpoints and grasping poses. A common strategy for generating a sampling distribution is to collect pose samples with motion capture data [14,4].

\section{1 libhand training set:}

To further examine the effect of training data, we created a massive custom training set of 25,000,000 RGB$\mathrm{D}$ training instances with the open-source libhand model. We modified the code to include a forearm and output depth data, semantic segmentations, and keypoint annotations. We emphasize that this synthetic training set is distinct from our new test dataset of real images.

Synthesis parameters: To avoid biasing our synthetic training set away from unlikely, but possible, poses we do not use motion capture data. Instead, we take a brute-force approach based on rejection-sampling. We

\begin{tabular}{|c|c|c|c|c|c|}
\hline Dataset & Generation & Viewpoint & Views & Size & Subj. \\
\hline ICL 45 & Real + manual annot. & 3rd Pers. & 1 & 331,000 & 10 \\
\hline NYU 47 & Real + auto annot. & 3rd Pers. & 3 & 72,757 & 1 \\
\hline UCI-EGO 35\| & Synthetic & Egocentric & 1 & 10,000 & 1 \\
libhand [50] & Synthetic & Generic & 1 & $25,000,000$ & 1 \\
\hline
\end{tabular}

Table 3 Training data sets: We broadly categorize training datasets by the method used to generate the data and annotations: real data + manual annotations, real data + automatic annotations, or synthetic data (and automatic annotations). Most existing datasets are viewpoint-specific (tuned for 3rd-person or egocentric recognition) and limited in size to tens of thousands of examples. NYU is unique in that it is a multiview dataset collected with multiple cameras, while ICL contains shape variation due to multiple (10) subjects. To explore the effect of training data, we use the public libhand animation package to generate a massive training set of 25 million examples.

uniformly and independently sample joint angles (from a bounded range), and throw away invalid samples that yield self-intersecting 3D hand poses. Specifically, using the libhand joint identifiers shown in figure 3 , we generate poses by uniformly sampling from bounded ranges, as shown in Table. 2

Quasi-Synthetic backgrounds: An under-emphasized aspect of synthetic training data is the choice of synthetic backgrounds. For methods operating on presegmented images [20,33,42, this is likely not an issue. However, for active hands "in-the-wild", the choice of synthetic backgrounds, surfaces, and interacting objects is likely important. Moreover, some systems require an explicit negative set (of images not containing hands) for training. To create such a background/negative training set, we take a quasi-synthetic approach by applying random affine transformations to 5,000 images of real scenes, yielding a total of $1,000,0000$ pseudo-synthetic backgrounds. We found it useful to include human bodies in the negative set because faces are common distractors for hand models.

\section{Methods}

Next we survey existing approaches to hand pose estimation (summarized in Table 4). We conclude by introducing a simple volumetric nearest-neighbor (NN) baseline.

\subsection{Taxonomy}

Trackers versus detectors: We focus our analysis on single-frame methods. For completeness, we also consider several tracking baselines [28,32,18] needing ground-truth initialization. Manual initialization may provide an unfair advantage, but we will show that 


\begin{tabular}{|c|c|c|c|c|}
\hline Description & Identifiers & bend & side & elongation \\
\hline Intermediate and Distal Joints & $F_{1: 4,2: 3}$ & $U\left(\frac{-\pi^{r}}{2}, \frac{\pi^{r}}{7}\right)$ & 0 & 0 \\
Proximal-Carpal Joints & $F_{1: 4,4}$ & $U\left(\frac{-\pi}{2} r, \frac{\pi}{7} r\right)$ & $U\left(\frac{-\pi}{8} r, \frac{\pi}{8}^{r}\right)$ & 0 \\
Thumb Metacarpal & $F_{5,4}$ & $U\left(-1^{r}, .5^{r}\right)$ & $U\left(-7^{r}, 1.2^{r}\right)$ & $U\left(.8^{r}, 1.2^{r}\right)$ \\
Thumb Proximal & $F_{5,3}$ & $U\left(-1^{r},-.6^{r}\right)$ & $U\left(-.2^{r}, .5^{r}\right)$ & 0 \\
Wrist Articulation & $P_{1}$ & $U\left(-1^{r}, 1^{r}\right)$ & $U\left(-.5^{r}, .8^{r}\right)$ & 0 \\
\hline
\end{tabular}

Table 2 Synthetic hand distribution: We render synthetic hands with joint angles sampled from the above uniform distributions. bend refers to the natural extension-retraction of the finger joints. The proximal-carpal, wrist and thumb joints are additionally capable of side-to-side articulation. We do not consider a third type of articulation, twist, because it would be extremely painful and result in injury. We model anatomical differences by elongating some bones fanning out from a joint. Additionally, we apply an isotropic global metric scale factor sampled from the range $U\left(\frac{2}{3}, \frac{3}{2}\right)$. Finally, we randomize the camera viewpoint by uniformly sampling tilt, yaw and roll from $U(0,2 \pi)$.

\begin{tabular}{|c|c|c|c|c|c|c|}
\hline Method & Approach & Model-drv. & Data-drv. & Detection & Implementation & FPS \\
\hline Simulate 23 & Tracker (simulation) & Yes & No & Initialization & Published & 50 \\
\hline NiTE2 32] & Tracker (pose search) & No & Yes & Initialization & Public & $>60$ \\
\hline Particle Swarm Opt. (PSO) 28] & Tracker (PSO) & Yes & No & Initialization & Public & 15 \\
\hline Hough Forest [51| & Decision forest & Yes & Yes & Decision forest & Ours & 12 \\
\hline Random Decision Forest (RDF) 20] & Decision forest & No & Yes & - & Ours & 8 \\
\hline Latent Regression Forest (LRF) 45] & Decision forest & No & Yes & - & Published & 62 \\
\hline DeepJoint [4] & Deep network & Yes & Yes & Decision forest & Published & 25 \\
\hline DeepPrior 26 & Deep network & No & Yes & Scanning window & Ours & 5000 \\
\hline DeepSegment 12] & Deep network & No & Yes & Scanning window & Ours & 5 \\
\hline Intel PXC [18] & Morphology (convex detection) & No & No & Heuristic segment & Public & $>60$ \\
\hline Cascades 35 & Hierarchical cascades & No & Yes & Scanning window & Provided & 30 \\
\hline EPM 53 & Deformable part model & No & Yes & Scanning window & Ours & $1 / 2$ \\
\hline Volumetric Exemplars & Nearest neighbor (NN) & No & Yes & Scanning volume & Ours & $1 / 15$ \\
\hline
\end{tabular}

Table 4 Summary of methods: We broadly categorize the pose estimation systems that we evaluate by their overall approach: decision forests, deep models, trackers, or others. Though we focus on single-frame systems, we also evaluate trackers by providing them manual initialization. Model-driven methods make use of articulated geometric models at test time, while data-driven models are trained beforehand on a training set. Many systems begin by detecting hands with a Hough-transform or a scanning window/volume search. Finally, we made use of public source code when available, or re-implemented the system ourselves, verifying our implementation's accuracy on published benchmarks. 'Published' indicates that published performance results were used for evaluation, while 'public' indicates that source code was available, allowing us to evaluate the method on additional testsets. We report the fastest speeds (in FPS), either reported or our implementation's.

single-frame methods are still nonetheless competitive, and in most cases, outperform tracking-based approaches. One reason is that single-frame methods essentially "reinitialize" themselves at each frame, while trackers cannot recover from an error.

Data-driven versus model-driven: Historic attempts to estimate hand pose optimized a geometric model to fit observed data [7, 1,43. Recently, Oikonomidis et al. 28] achieved success using GPU accelerated Particle Swarm Optimization. However, such optimizations remain notoriously difficult due to local minima in the objective function. As a result, model driven systems have found their successes mostly to the tracking domain, where initialization constrains the search space [42,23, 33. For single image detection, various fast classifiers [20, 18] have obtained real-time speeds. Most of the systems we evaluate fall into this category. When these classifiers are trained with data synthesized from a geometric model, they can be seen as efficiently approximating model fitting.

Multi-stage pipelines: It is common to treat the initial detection (candidate generation) stage as separate from hand-pose estimation. Some systems use special purpose detectors as a "pre-processing" stage 51,20, 47, 18, 28, 16, 5, 36. Others use a geometric model for inverse-kinematic (IK) refinement/validation during a "post-processing" stage [51,47,23,42. A segmentation pre-processing stage has been historically popular. Typically, the depth image is segmented with simple morphological operations 31 or the RGB image is segmented with skin classifiers [49]. allowing features such as Zernike moments [5] or skeletonizations 31] to be computed. The latter appears difficult to generalize across subjects and scenes with varying lighting [33. We evaluate a depth-based segmentation system [18] for completeness.

\subsection{Architectures}

In this section, we describe popular architectures for hand-pose estimation, placing in bold those systems that we empirically evaluate.

Decision forests: Decision forests constitute a dominant paradigm for estimating hand pose from depth. 
Hough Forests [51] take a two-stage approach of hand detection followed by pose estimation. Random Decision Forests (RDFs) 20] and Latent Regression Forests (LRFs) 45] leave the initial detection stage unspecified, but both make use of coarse-to-fine decision trees that perform rough viewpoint classification followed by detailed pose estimation. We experimented with several detection front-ends for RDFs and LRFs, finally selecting the first-stage detector from Hough Forests for its strong performance.

Part model: Pictorial structure models have been popular in human body pose estimation [52, but they appear rare in hand pose estimation. For completeness, we evaluate a deformable part model defined on depth image patches 15. We specifically train an exemplar part model (EPM) constrained to model deformations consistent with 3D exemplars [53], which will be described further in a tech report.

Deep models: Recent systems have explored the use of deep neural nets for hand pose estimation. We consider three variants in our experiments. DeepJoint [47] uses a three stage pipeline that initially detects hands with a decision forest, regresses joint locations with a deep network, and finally refines joint predictions with inverse kinematics (IK). DeepPrior [26] is based on a similar deep network, but does not require an IK stage and instead relies on the network itself to learn a spatial prior. DeepSeg 12 takes a pixel-labeling approach, predicting joint labels for each pixel, followed by a clustering stage to produce joint locations. This procedure is reminiscent of pixel-level part classification of Kinect [40], but substitutes a deep network for a decision forest.

\subsection{Volumetric exemplars}

We propose a nearest-neighbor (NN) baseline for additional diagnostic analysis. Specifically, we convert depth map measurements into a 3D voxel grid, and simultaneously detect and estimate pose by scanning over this grid with volumetric exemplar templates.

Voxel grid: Depth cameras report depth as a function of pixel $(u, v)$ coordinates: $D(u, v)$. To construct a voxel grid, we first re-project these image measurements into $3 \mathrm{D}$ using known camera intrinsics $f_{u}, f_{v}$.

$(x, y, z)=\left(\frac{u}{f_{u}} D(u, v), \frac{v}{f_{v}} D(u, v), D(u, v)\right)$

Given a test depth image, we construct a binary voxel grid $V[x, y, z]$ that is ' 1 ' if a depth value is observed at a quantized $(x, y, z)$ location. To cover the rough viewable region of a camera, we define a coordinate frame of $M^{3}$ voxels, where $M=200$ and each voxel spans $10 \mathrm{~mm}^{3}$. We similarly convert training examples into volumetric exemplars $E[x, y, z]$, but instead use a smaller $N^{3}$ grid of voxels (where $N=30$ ), consistent with the size of a hand.

Occlusions: When a depth measurement is observed at a position $\left(x^{\prime}, y^{\prime}, z^{\prime}\right)=1$, all voxels behind it are occluded $z>z^{\prime}$. We define occluded voxels to be ' 1 ' for both the test-time volume $V$ and training exemplar $E$.

Distance measure: Let $V_{j}$ be the $j^{\text {th }}$ subvolume (of size $N^{3}$ ) extracted from $V$, and let $E_{i}$ be the $i^{\text {th }}$ exemplar. We simultaneously detect and estimate pose by computing the best match in terms of Hamming distance:

$$
\begin{aligned}
\left(i^{*}, j^{*}\right) & =\underset{i, j}{\operatorname{argmin}} \operatorname{Dist}\left(E_{i}, V_{j}\right) \quad \text { where } \\
\operatorname{Dist}\left(E_{i}, V_{j}\right) & =\sum_{x, y, z} \mathcal{I}\left(E_{i}[x, y, z] \neq V_{j}[x, y, z]\right),
\end{aligned}
$$

such that $i^{*}$ is the best-matching training exemplar and $j^{*}$ is its detected position.

Efficient search: A naive search over exemplars and subvolumes is prohibitively slow. But because the underlying features are binary and sparse, there exist considerable opportunities for speedup. We outline two simple strategies. First, one can eliminate subvolumes that are empty, fully occluded, or out of the camera's field-of-view. Song et al. 41] refer to such pruning strategies as "jumping window" searches. Second, one can compute volumetric Hamming distances with 2D computations:

$$
\begin{aligned}
& \operatorname{Dist}\left(E_{i}, V_{j}\right)=\sum_{x, y}\left|e_{i}[x, y]-v_{j}[x, y]\right| \quad \text { where } \\
& e_{i}[x, y]=\sum_{z} E_{i}[x, y, z], \quad v_{j}[x, y]=\sum_{z} V_{j}[x, y, z]
\end{aligned}
$$

Intuitively, because our 3D volumes are projections of 2.5D measurements, they can be sparsely encoded with a 2D array (see Fig. 4). Taken together, our two simple strategies imply that a $3 \mathrm{D}$ volumetric search can be made as practically efficient as a $2 \mathrm{D}$ scanning-window search. For a modest number of exemplars, our implementation still took tens of seconds per frame, which sufficed for our offline analysis. We posit faster NN algorithms could produce real-time performance [24,25]. 


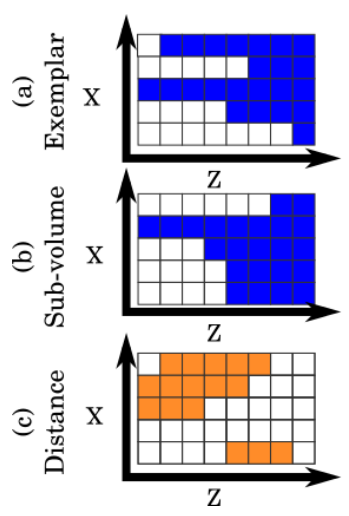

Fig. 4 Volumetric Hamming distance: We visualize $3 \mathrm{D}$ voxels corresponding to an exemplar (a) and subvolume (b). For simplicity, we visualize a $2 \mathrm{D}$ slice along a fixed y-value. Because occluded voxels are defined to be ' 1 ' (indicating they are occupied, shown in blue) the total Hamming distance is readily computed by the L1 distance between projections along the z-axis (c), mathematically shown in Eq.4.

Fig. 5 Windows v. volumes: 2D scanning windows (a) versus $3 \mathrm{D}$ scanning volumes (b). Volumes can ignore background clutter that lie outside the 3D scanning volume but still fall inside its 2D projection. For example, when scoring the above hand, a 3D scanning volume will ignore depth measurements from the shoulder and head, unlike a $2 \mathrm{D}$ scanning window. (a)

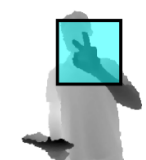

(b)

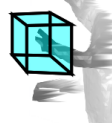

Comparison: Our volumetric exemplar baseline uses a scanning volume search and $2 \mathrm{D}$ depth encodings. It is useful to contrast this with a "standard" 2D scanningwindow template on depth features [19. First, our exemplars are defined in metric coordinates (Eq. 11). This means that they will not fire on the small hands of a toy figurine, unlike a scanning window search over scales. Second, our volumetric search ensures that the depth encoding from a local window contain features only within a fixed $N^{3}$ volume. This gives it the ability to segment out background clutter, unlike a $2 \mathrm{D}$ window (Fig. 5).

\section{Protocols}

\subsection{Evaluation}

Reprojection error: Following past work, we evaluate pose estimation as a regression task that predicts a set of 3D joint locations [45, 28, 33, 46, 20]. Given a predicted and ground-truth pose, we compute both the average and max 3D reprojection error (in $\mathrm{mm}$ ) across all joints. We use the skeletal joints defined by libhand [50]. We then summarize performance by plotting the proportion of test frames whose average (or max) error falls below a threshold.

Error thresholds: Much past work considers performance at fairly low error thresholds, approaching $10 \mathrm{~mm}$ 45, 47,51]. Interestingly, [26] show that established benchmarks such as the ICL testset include annotation errors of above $10 \mathrm{~mm}$ in over a third of their

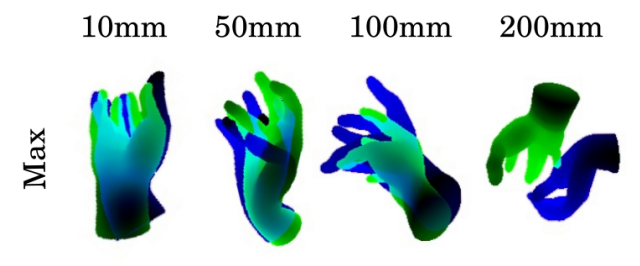

Fig. 6 Our error criteria: For each predicted hand, we calculate the average and maximum distance (in $\mathrm{mm}$ ) between its skeletal joints and a ground-truth. In our experimental results, we plot the fraction of predictions that lie within a distance threshold, for various thresholds. This figure visually illustrates the misalignment associated with various thresholds for max error. A 50mm max-error seems visually consistent with a "roughly correct pose estimation", and a $100 \mathrm{~mm}$ max-error is consistent with a "correct hand detection".

frames. Ambiguities arise from manual labeling of joints versus bones and centroids versus surface points. We rigorously evaluate human-level performance through inter-annotator agreement on our new testset (Fig. 14). Overall, we find that max-errors of $20 \mathrm{~mm}$ approach the limit of human accuracy for closeby hands. We present a qualitative visualization of max error at different thresholds in Fig. 6. 50 $\mathbf{6 m}$ appears consistent with a roughly correct pose, while an error within $100 \mathrm{~mm}$ appears consistent with a correct detection. Our qualitative analysis is consistent with empirical studies of human grasp [2] and gesture [4] which also suggest that $50 \mathrm{~mm}$ is sufficient to capture difference in gesture or grasp. For completeness, we plot results across a large range of thresholds, but highlight 50 and $100 \mathrm{~mm}$ thresholds for additional analysis.

Detection issues: Reprojection error is hard to define during detection failures: that is, false positive hand detections or missed hand detections. Such failures are likely in cluttered scenes or when considering scenes containing zero or two hands. If a method produced zero detections when a hand was present, or produced one if no hand was present, this was treated as a "maxedout" reprojection error (of $\infty \mathrm{mm}$ ). If two hands were present, we scored each method against both and took the minimum error. Though we plan to release our evaluation software, we give pseudocode in Alg. 1 .

Missing data: Another challenge with reprojection error is missing data. First, some methods predict 2D rather than 3D joints 18,31,47,12. Inferring depth should in theory be straightforward with Eq. 1, but small 2D errors in the estimated joint can cause significant errors in the estimated depth. We report back the centroid depth of a segmented/detected hand if the measured depth lies outside the segmented volume. 


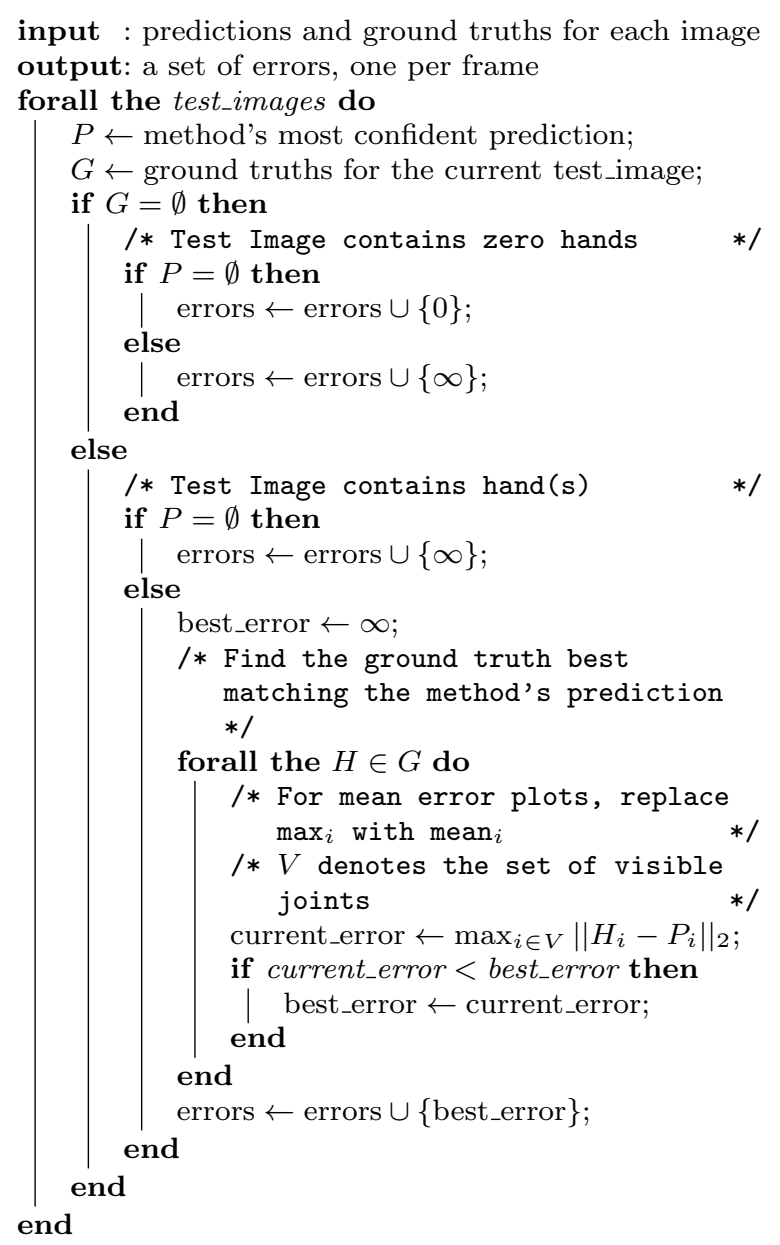

Algorithm 1: Scoring Procedure: For each frame we compute a max or mean re-projection error for the ground truth(s) $G$ and prediction(s) $P$. We later plot the proportion of frames with an error below a threshold, for various thresholds.

Past comparisons appear not to do this [26, somewhat unfairly penalizing 2D approaches 47. Second, some methods may predict a subset of joints [18,31. To ensure a consistent comparison, we force such methods to predict the locations of visible joints with a postprocessing inverse-kinematics (IK) stage 47. We fit the libhand kinematic model to the predicted joints, and infer the location of missing ones. Third, ground-truth joints may be occluded. By convention, we only evaluate visible joints in our benchmark analysis.

Implementations: We use public code when available 28, 32, 18. Some authors responded to our request for their code 35. When software was not available, we attempted to re-implement methods ourselves. We were able to successfully reimplement [26,51,20, matching the accuracy on published results [45,26]. In other cases, our in-house implementations did not suffice [47,
45. For these latter cases, we include published performance reports, but unfortunately, they are limited to their own datasets. This partly motivated us to perform a multi-dataset analysis. In particular, previous benchmarks have shown that one can still compare algorithms across datasets using head-to-head matchups (similar to approaches used to rank sports teams that do not directly compete 29]). We use our NN baseline to do precisely this. Finally, to spur further progress, we will make all implementations publicly available, together with our evaluation code.

\subsection{Annotation}

We now describe how we collect ground truth annotations. We present the annotator with cropped RGB and Depth images. They then click semantic key-points, corresponding to specific joints, on either the RGB or Depth images. To ease the annotator's task and to get 3D keypoints from $2 \mathrm{D}$ clicks we invert the forward rendering (graphics) hand model provided by libhand which projects model parameters $\theta$ to $2 \mathrm{D}$ keypoints $P(\theta)$. While they label joints, an inverse kinematic solver minimizes the distance between the currently annotated $2 \mathrm{D}$ joint labels, $\forall_{j \in J} L_{j}$, and those projected from the libhand model parameters, $\forall_{j \in J} P_{j}(\theta)$.

$\min _{\theta} \sum_{j \in J}\left\|L_{j}-P_{j}(\theta)\right\|_{2}$

The currently fitted libhand model, shown to the annotator, updates online as more joints are labeled. When the annotator indicates satisfaction with the fitted model, we proceed to the next frame. We give an example of the annotation process in figure 7.

Strengths: Our annotation process has several strengths. First, kinematic constraints prevent some possible combination of keypoints: so it is often possible to fit the model by labeling only a subset of keypoints. Second, the fitted model provides annotations for occluded keypoints. Third and most importantly, the fitted model provides 3D (x,y,z) keypoint locations given only $2 \mathrm{D}(\mathrm{u}, \mathrm{v})$ annotations.

Disagreements: As shown in in Fig. 14, annotators disagree substantially on the hand pose, in a surprising number of cases. In applications, such as sign language [44] ambiguous poses are typically avoided. We believe it is important to acknowledge that, in general, it may not be possible to achieve full precision. Figure 8 illustrates two examples of these annotator disagreements. 


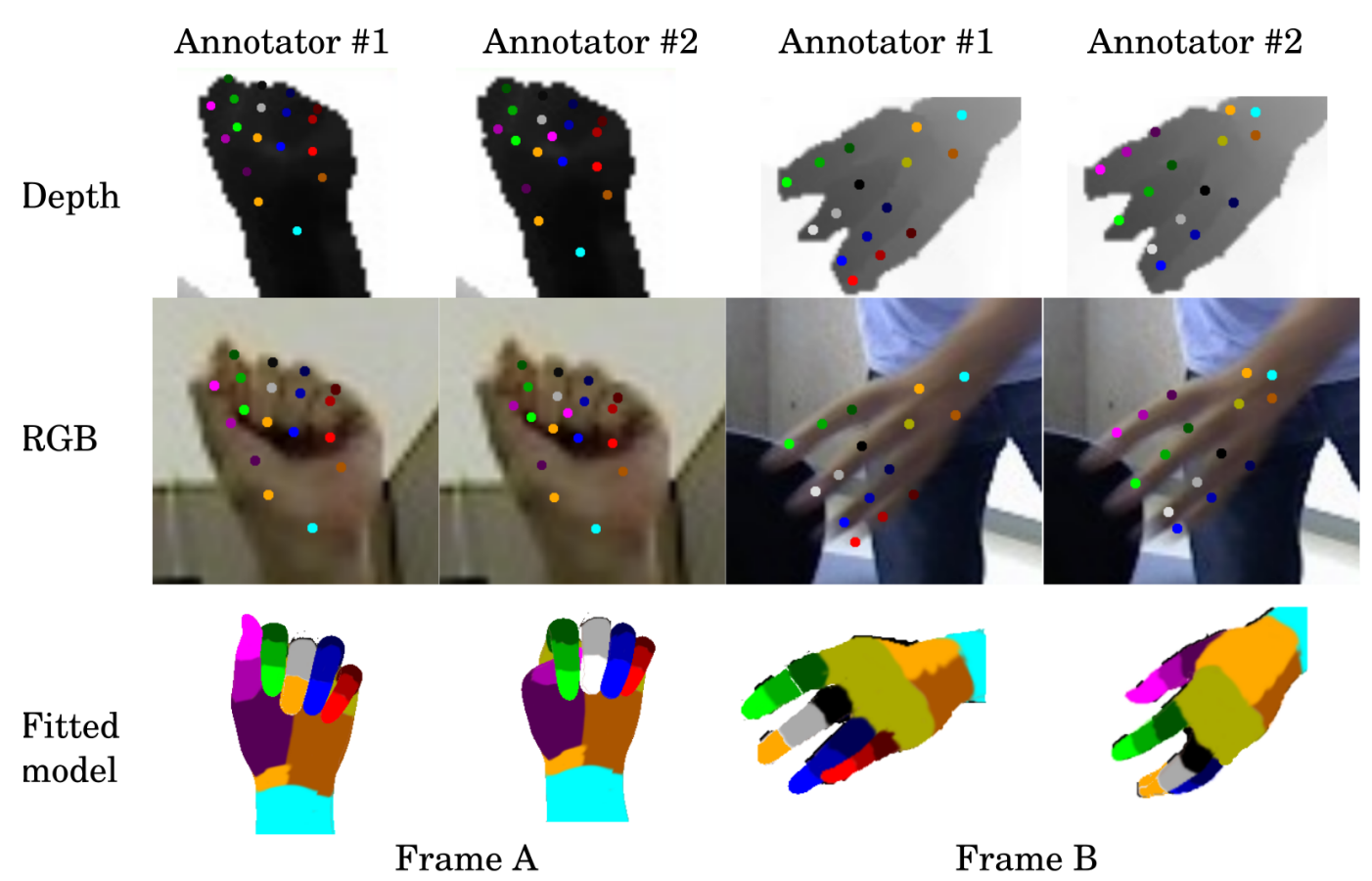

Fig. 8 Annotator disagreements: With whom do you agree? We show two frames where annotators disagreed. The top two rows show the RGB and depth images presented and the keypoint annotations received from the annotator. The bottom row shows the libhand model fitted to those keypoint annotations.

In Frame A, the confusion revolves about the thumb position. Is the thumb occluded, folded down behind the other digits, or does it stand upright? The resolution, in both color and depth makes this hard to decide. Long range (low resolution) scenarios are important; But in these scenarios we cannot expect performance comparable to that found in near range.

Similarly, in Frame $B$ one finger is occluded, but which one? Annotator 1 believes the thumb is occluded. Annotator 2 believes the pinky is occluded. The fitted libhand models show that either interpretation is plausible. In this author's opinion, annotator 1 is more consistent with the RGB evidence while annotator 2 is more consistent with the Depth evidence.

\section{RGB Depth LibHand}

(A)

(B)
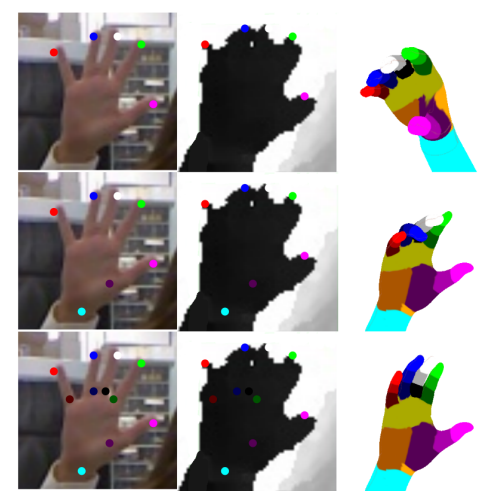

Fig. 7 Annotation procedure: We annotate until we are satisfied that the fitted hand pose matches the RGB and Depth data. The first two columns show the image evidence presented and keypoints received. The right most column shows the fitted libhand model. (A) the IK solver is able to easily fit a model to the five given keypoints, but it doesn't match the image well. (B) The annotator attempts to correct the model, to better match the image, by labeling the wrist. (C) Labeling additional finger joints finally yields and acceptable solution.

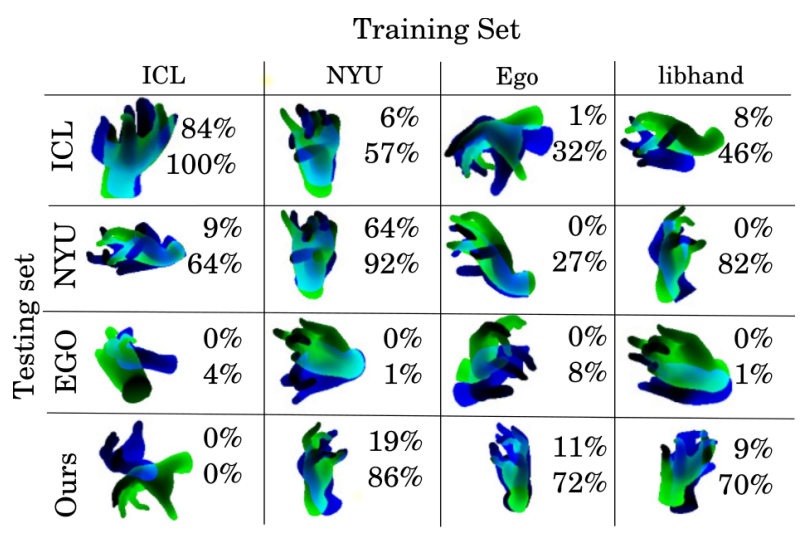

Table 5 Cross-dataset generalization: We compare training and test sets using a 1-NN classifier. Diagonal entries represent the performance using corresponding train and test sets. In each grid entry, we denote the percentage of test frames that are correct $(50 \mathrm{~mm}$ max-error, above, and $50 \mathrm{~mm}$ average-error, below) and visualize the median error using the colored overlays from Fig. 6. We account for sensor specific noise artifacts using established techniques 3 . Please refer to the text for more details. 

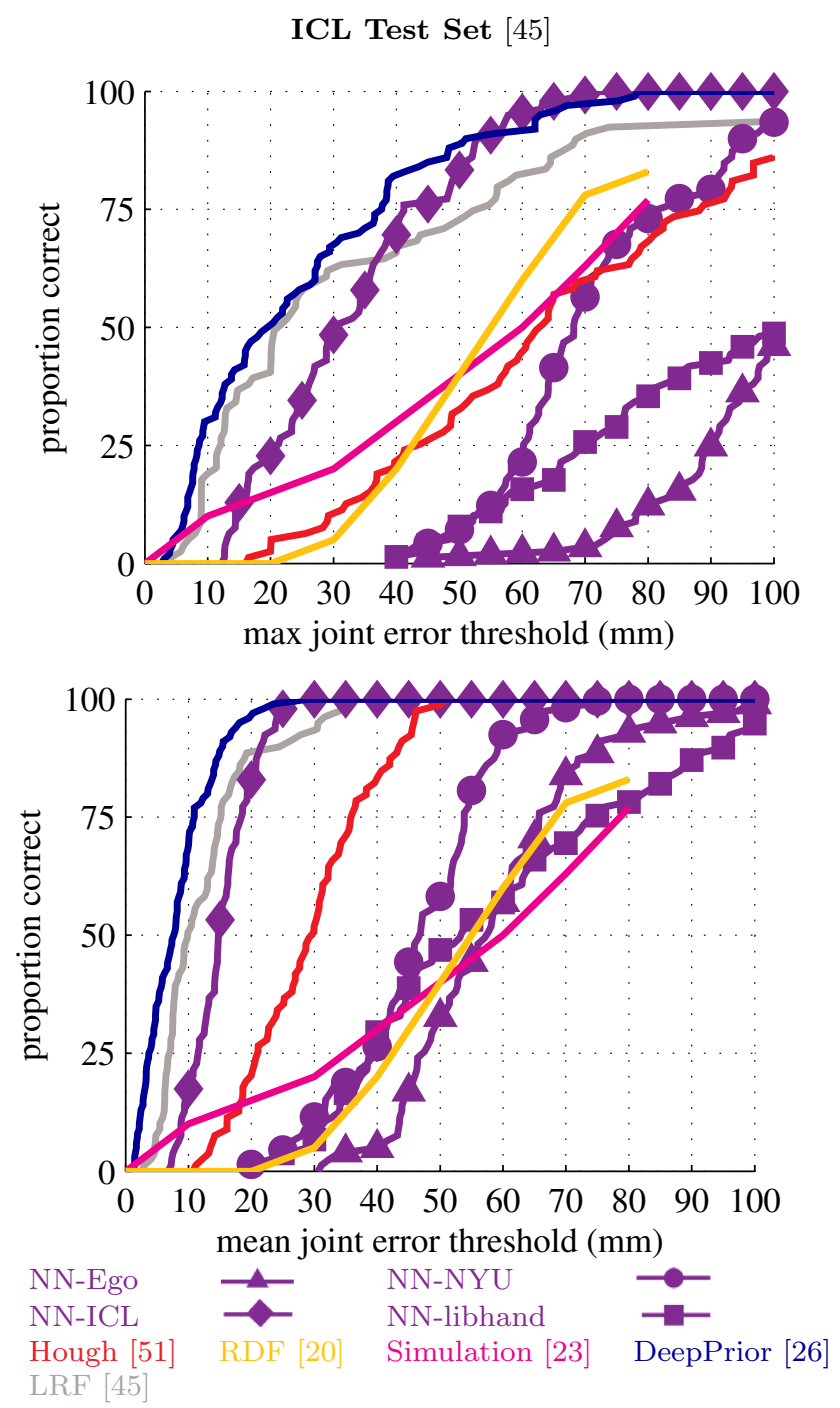

Fig. 9 We plot results for several systems on the ICL testset using max-error (top) and average-error (bottom). Except for 1-NN, all systems are trained on the corresponding train set (in this case ICL-Train). To examine cross-dataset generalization, we also plot the performance of our NN-baseline constructed using alternate sets (NYU, EGO, and libhand). When trained with ICL, NN performs as well or better than prior art. One can find near-perfect pose matches in the training set (see Fig. 11). Please see text for further discussion.

\section{Results}

We now report our experimental results, comparing datasets and methods. We first address the "state of the problem": what aspects of the problem have been solved, and what remain open research questions? We conclude by discussing the specific lessons we learned and suggesting directions for future systems.

Mostly-solved (distinct poses): Fig. 9 shows that hand pose estimation is mostly solved on datasets of uncluttered scenes where hands face the camera (i.e. ICL).

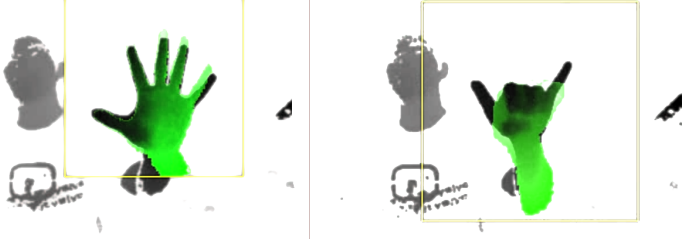

Fig. 10 Min vs max error: Compared to state-of-theart, our 1-NN baseline often does relatively better under the average-error criterion than under the max-error criterion. When it can find (nearly) an exact match between training and test data (left) it obtains very low error. However, it does not generalize well to unseen poses (right). When presented with a new pose it will often place some fingers perfectly but others totally wrong. The result is a reasonable mean error but a high max error.

Deep models, decision forests, and NN all perform quite well, both in terms of articulated pose estimation (85\% of frames are within 50mm max-error) and hand detection (100\% are within $100 \mathrm{~mm}$ max-error). Surprisingly, NN outperforms decision forests by a bit. However, when $\mathrm{NN}$ is trained on other datasets with larger pose variation, performance is considerably worse. This suggests that the test poses remarkably resemble the training poses. But, this may be reasonable for applications targeting sufficiently distinct poses from a finite vocabulary (e.g., a gaming interface). These results suggest that the state-of-the-art accurately predicts distinct poses (i.e.50 $\mathrm{mm}$ apart) in uncluttered scenes.

Major progress (unconstrained poses): The NYU testset still considers isolated hands, but includes a wider range of poses, viewpoints, and subjects compared to ICL (see Fig. 2). Fig. 12 reveals that deep models perform the best for both articulated pose estimation $(96 \%$ accuracy) and hand detection (100\% accuracy). While decision forests struggle with the added variation in pose and viewpoint, NN still does quite well. In fact, when measured with average (rather than max) error, NN nearly matches the performance of [47]. This suggests that exemplars get most, but not all fingers, correct (see Fig. 6). Overall, we see noticeable progress on unconstrained pose estimation since 2007 [10].

Unsolved (low-res, objects, occlusions, clutter): When considering datasets (Fig. 14 and 15) with distant (lowres) hands and background clutter due to objects or interacting surfaces (Fig. 6), results are significantly worse. Note that many applications [40] often demand hands to lie at distances greater than $750 \mathrm{~mm}$. For such scenes, hand detection is still a challenge. Scanning window approaches (such as our NN baseline) tend to outperform multistage pipelines [20,12, which may make 


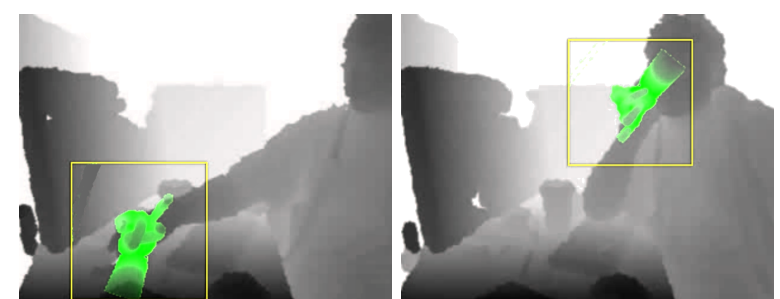

Fig. 11 Complex backgrounds: Most existing systems, including our own 1-NN baseline, fail when challenged with complex backgrounds which cannot be trivially segmented. These backgrounds significantly alter the features extracted and processed and thus prevent even the best models from producing sensible output.

\section{NYU Test Dataset 47}
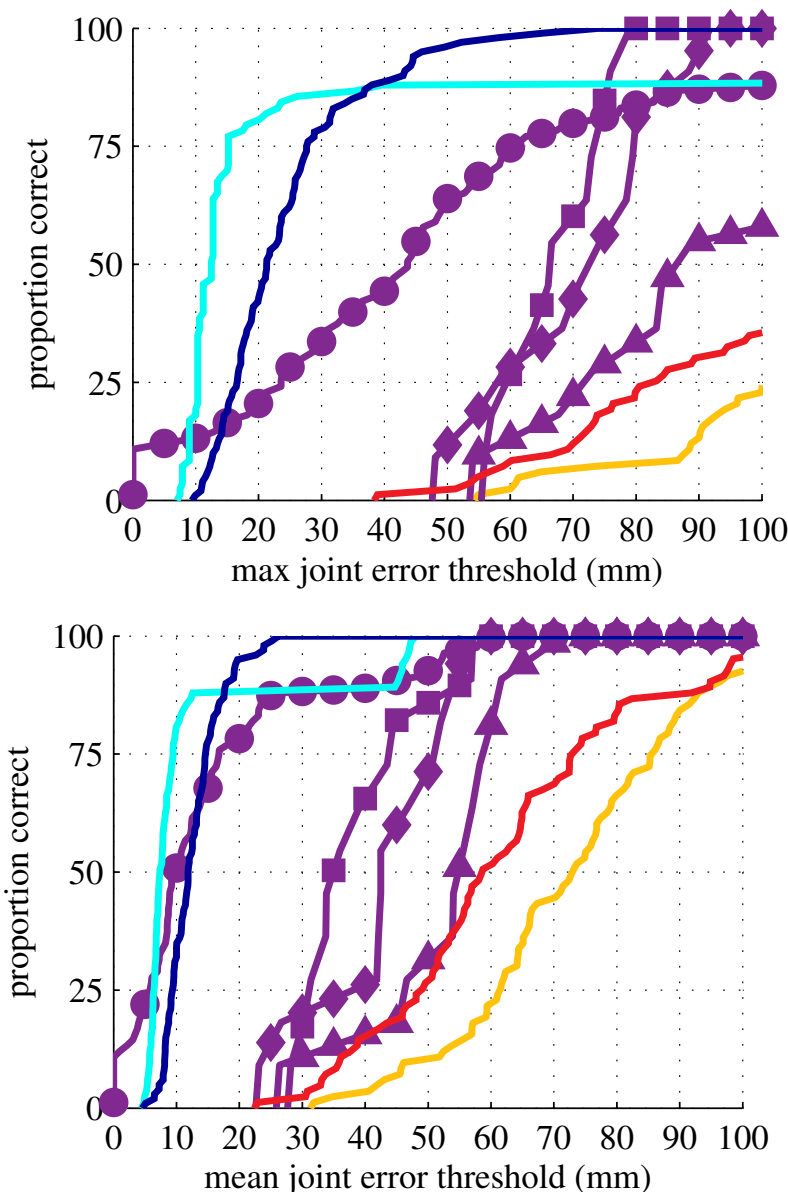

NN-Ego

NN-ICL

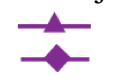

NN-NYU

NN-libhand

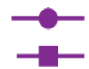

Hough 51]

DeepJoint 47

DeepPrior [26]

Fig. 12 Deep models [4, 26] perform noticeably better than other systems, and appear to solve both articulated pose estimation and hand detection for uncluttered single-user scenes (common in the NYU testset). However, the other systems compare more favorably under average error. In Fig. 6, we interpret this disconnect by using $1-\mathrm{NN}$ to show that each test hand commonly matches a training example in all but one finger. Please see text for further discussion. (a) Latent Hough Detection

(c) per-pixel classification

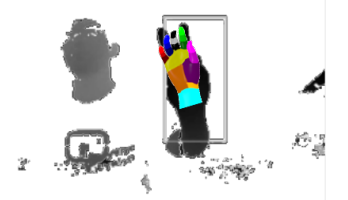

(b) Hough orientation failure

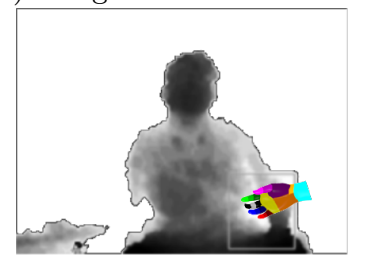

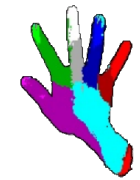

(d) hard segmentation

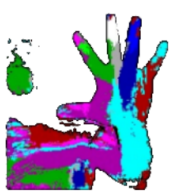

Fig. 13 Many approach the problem of hand pose estimation in three phases: (1) detect and segment (2) estimate pose (3) validate or refine [51,20, 47, 45, 18. However, when an earlier stage fails, the later stages are often unable to recover. When detection and segmentation are non-trivial, this becomes to root cause of many failures. For example, Hough forests [51] (a) first estimate the hand's location and orientation. They then convert to a cardinal translation and rotation before estimating joint locations. (b) When this first stage fails, the second stage cannot recover. (c) Other methods assume that segmentation is solved [20]12], (d) when background clutter is inadvertently included by the hand segmenter, the finger pose estimator is prone to spurious outputs.

an unrecoverable error in the first (detection and segmentation) stage. We show some illustrative examples in Fig. 13. However, overall performance is still lacking, particularly when compared to human performance. Though interestingly, human (annotator) accuracy also degrades for low-resolution hands far away from the camera (Fig. 14). Our results suggest that scenes of inthe-wild hand activity are still beyond the reach of the state-of-the-art.

Training data: We use our NN-baseline to analyze the effect of training data in Table 5. Our NN model performed better using the NYU training set [47. (consisting of real data automatically labeled with a geometrically-fit 3D CAD model) than with the libhand training set. While performance increases by enlarging the synthetic training set (Fig. 16), this quickly becomes intractable. This reflects the difficulty in using synthetic data: one must carefully model priors [26], sensor noise, [17] and hand shape variations between users 46]. Moreover, in some cases, the variation in the performance of NN (dependent on the particular training set) exceeded the variation between model architectures (decision forests versus deep models) - Fig. 9. Our results suggest the diversity and realism of the training set is as important than the model form learned from $i t$. 

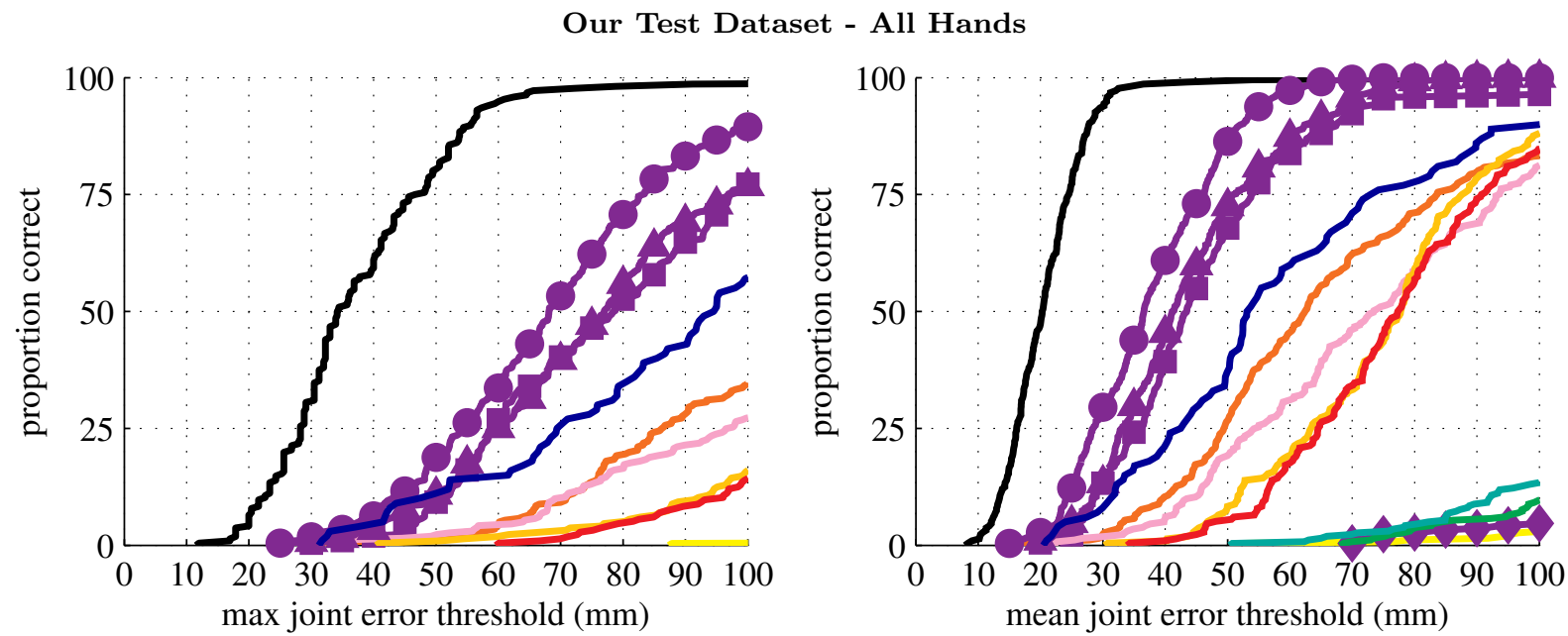

Our Test Dataset - Near Hands $(\leq 750 \mathrm{~mm})$
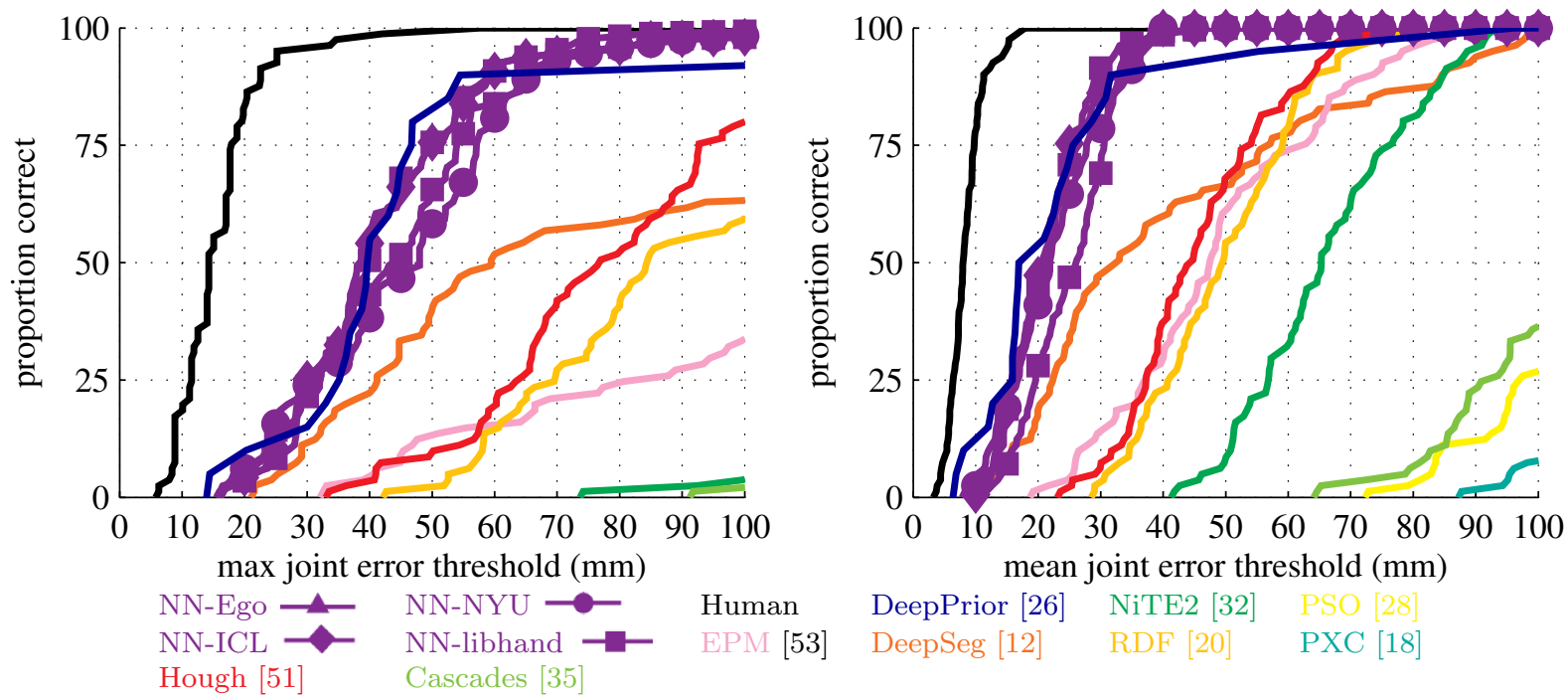

Fig. 14 We designed our dataset to address the remaining challenges of in "in-the-wild" hand pose estimation, including scenes with low-res hands, clutter, object/surface interactions, and occlusions. We plot human-level performance (as measured through inter-annotator agreement) in black. On nearby hands (within $750 \mathrm{~mm}$, as commonly assumed in prior work) our annotation quality is similar to existing testsets such as ICL 26]. This is impressive given that our testset includes comparatively more ambiguous poses (see Sec. 5.2. Our dataset includes far away hands, for which even humans struggle to accurately label. Moreoever, several methods (Cascades,PXC,NiTE2,PSO) fail to correctly localize any hand at any distance, though the meanerror plots are more forgiving than the max-error above. In general, NN-exemplars and DeepPrior perform the best, correctly estimating pose on $75 \%$ of frames with nearby hands.

NN vs Deep models: Overall, our 1-NN baseline proved to be suprisingly strong, outperforming or matching the performance of most prior systems. This holds true even for moderately-sized training sets with tens of thousands of examples, suggesting that much prior work essentially memorizes training examples. One contribution of our analysis is the notion that $N N$-exemplars provides a vital baseline for understanding the behavior of a proposed system in relation to its training set. In fact, DeepJoint 47] and DeepPrior 26] were the sole approaches to significantly outperform 1-NN (Figs. 9 and 12. This indicates that deep architectures gener- alize well to novel test poses. This may contrast with existing folk wisdom about deep models: that the need for large training sets suggests that these models essentially memorize. Our results indicate otherwise.

Conclusion: The past several years have shown tremendous progress regarding hand pose: training sets, testing sets, and models. Some applications, such as gaming interfaces and sign-language recognition, appear to be well-within reach for current systems. Less than a decade ago, this was not true [31,10,5]. Thus, we have made progress! But, challenges remain nonetheless. Specifically, when segmentation is hard due to ac- 

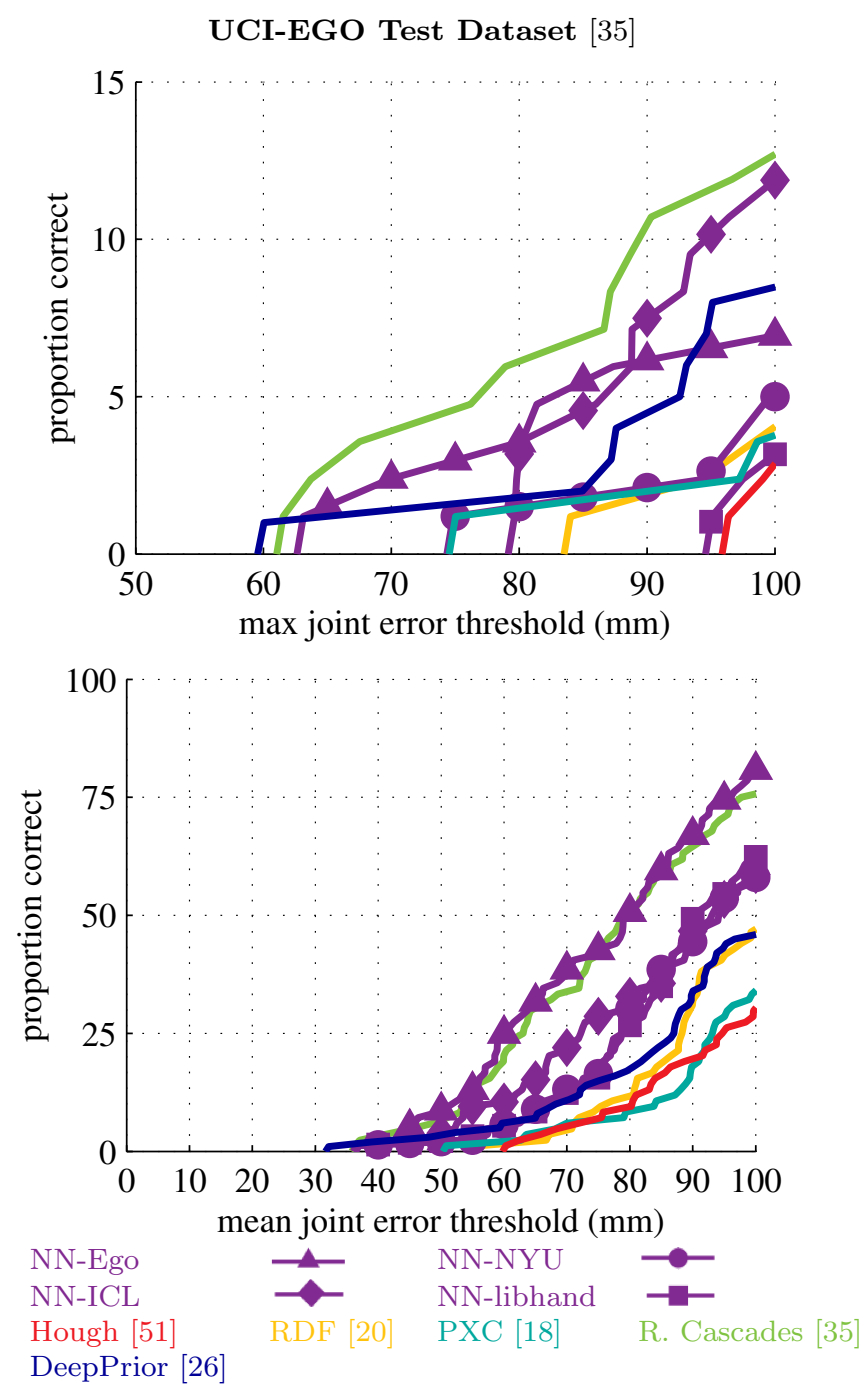

Fig. 15 For UCI-EGO, randomized cascades and our NN baseline do about as well, but overall, performance is considerably worse than other datasets. No methods are able to correctly estimate the pose (within $50 \mathrm{~mm}$ ) on any frames. Egocentric scenes contain more background clutter and object/surface interfaces, making even hand detection challenging for many methods.

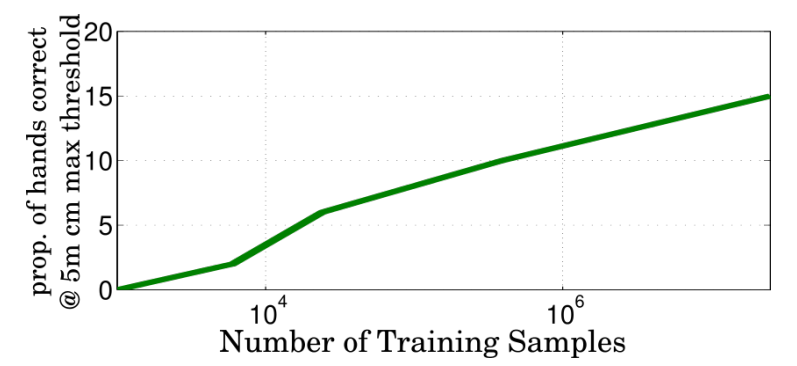

Fig. 16 Synthetic data vs. accuracy: Synthetic training set size impacts performance on our test testset. Performance grows logarithmically with the dataset size. Synthesis is theoretically unlimited, but practically becomes unattractively slow. tive hands or clutter, many existing methods fail. To illustrate these realistic challenges we introduce a novel testset. We demonstrate that realism and diversity in training sets is crucial, and can be as important as the choice of model architecture. In terms of model architecture, we perform a broad benchmark evaluation and find that deep models appear particularly well-suited for pose estimation. Finally, we demonstrate that NN using volumetric exemplars provides a startlingly potent baseline, providing an additional tool for analyzing both methods and datasets.

\section{References}

1. M. Bray, E. Koller-Meier, P. Müller, L. Van Gool, and N. N. Schraudolph. 3D hand tracking by rapid stochastic gradient descent using a skinning model. In In 1st European Conference on Visual Media Production (CVMP), 2004.

2. I. M. Bullock, S. Member, J. Z. Zheng, S. D. L. Rosa, C. Guertler, and A. M. Dollar. Grasp Frequency and Usage in Daily Household and Machine Shop Tasks. Haptics, IEEE Transactions on, 6(3):296-308, 2013.

3. M. Camplani and L. Salgado. Efficient spatio-temporal hole filling strategy for kinect depth maps. In Proceedings of SPIE, volume 8920, 2012.

4. C. Castellini, T. Tommasi, N. Noceti, F. Odone, and B. Caputo. Using object affordances to improve object recognition. Autonomous Mental Development, IEEE Transactions on, 3(3):207-215, 2011.

5. H. Cooper. Sign Language Recognition using Sub-Units. The Journal of Machine Learning Research, 13:22052231, 2012.

6. T. Y. D. Tang and T.-K. Kim. Real-time articulated hand pose estimation using semi-supervised transductive regression forests. In $I C C V$, pages 1-8, 2013.

7. Q. Delamarre and O. Faugeras. 3D Articulated Models and Multiview Tracking with Physical Forces. Computer Vision and Image Understanding, 81(3):328-357, Mar. 2001.

8. J. Deng, W. Dong, R. Socher, L.-J. Li, K. Li, and L. Fei-Fei. Imagenet: A large-scale hierarchical image database. In Computer Vision and Pattern Recognition, 2009. CVPR 2009. IEEE Conference on, pages 248-255. IEEE, 2009.

9. P. Dollar, C. Wojek, B. Schiele, and P. Perona. Pedestrian detection: An evaluation of the state of the art. Pattern Analysis and Machine Intelligence, IEEE Transactions on, 34(4):743-761, 2012.

10. A. Erol, G. Bebis, M. Nicolescu, R. D. Boyle, and X. Twombly. Vision-based hand pose estimation: A review. Computer Vision and Image Understanding, 108(1-2):52-73, Oct. 2007.

11. M. Everingham, L. Van Gool, C. K. Williams, J. Winn, and A. Zisserman. The PASCAL visual object classes (VOC) challenge. International journal of computer vision, 88(2):303-338, 2010.

12. C. Farabet, C. Couprie, L. Najman, and Y. LeCun. Learning hierarchical features for scene labeling. Pattern Analysis and Machine Intelligence, IEEE Transactions on, 35(8):1915-1929, 2013.

13. L. Fei-Fei, R. Fergus, and P. Perona. Learning generative visual models from few training examples: An incremental Bayesian approach tested on 101 object categories. 
Computer Vision and Image Understanding, 106(1):5970, Apr. 2007.

14. T. Feix, J. Romero, C. H. Ek, H. Schmiedmayer, and D. Kragic. A Metric for Comparing the Anthropomorphic Motion Capability of Artificial Hands. Robotics, IEEE Transactions on, 29(1):82-93, Feb. 2013.

15. P. F. Felzenszwalb, R. B. Girshick, D. McAllester, and D. Ramanan. Object detection with discriminatively trained part-based models. Pattern Analysis and $M a-$ chine Intelligence, IEEE Transactions on, 32(9):1627$1645,2010$.

16. M. Girard and A. A. Maciejewski. Computational Modeling for the Computer Animation of Legged Figures. ACM SIGGRAPH Computer Graphics, 19(3):263-270, 1985.

17. S. Gupta, R. Girshick, P. Arbeláez, and J. Malik. Learning rich features from rgb-d images for object detection and segmentation. In Computer Vision-ECCV 2014 , pages 345-360. Springer, 2014.

18. Intel. Perceptual computing SDK, 2013.

19. A. Janoch, S. Karayev, Y. Jia, J. T. Barron, M. Fritz, K. Saenko, and T. Darrell. A category-level 3d object dataset: Putting the kinect to work. In Consumer Depth Cameras for Computer Vision, pages 141-165. Springer London, 2013.

20. C. Keskin, F. Kıraç, Y. E. Kara, and L. Akarun. Hand pose estimation and hand shape classification using multi-layered randomized decision forests. In $E C C V$ 2012, pages 852-863. 2012.

21. C. Li and K. M. Kitani. Pixel-Level Hand Detection in Ego-centric Videos. 2013 IEEE Conference on Computer Vision and Pattern Recognition, pages 3570-3577, June 2013

22. D. R. Martin, C. C. Fowlkes, and J. Malik. Learning to detect natural image boundaries using local brightness, color, and texture cues. Pattern Analysis and Machine Intelligence, IEEE Transactions on, 26(5):530-549, 2004.

23. S. Melax, L. Keselman, and S. Orsten. Dynamics based 3D skeletal hand tracking. Proceedings of the ACM SIGGRAPH Symposium on Interactive 3D Graphics and Games - I3D '13, page 184, 2013.

24. A. Moore, A. Connolly, C. Genovese, A. Gray, L. Grone, N. Kanidoris II, R. Nichol, J. Schneider, A. Szalay, I. Szapudi, and L. Wasserman. Fast Algorithms and Efficient Statistics: N-point Correlation Functions. Dec. 2000.

25. M. Muja and D. G. Lowe. Scalable Nearest Neighbor Algorithms for High Dimensional Data. IEEE Transactions on Pattern Analysis and Machine Intelligence, 36(11):2227-2240, Nov. 2014

26. M. Oberweger, P. Wohlhart, and V. Lepetit. Hands Deep in Deep Learning for Hand Pose Estimation. Computer Vision Winter Workshop (CVWW), 2015.

27. E. Ohn-Bar and M. M. Trivedi. Hand Gesture Recognition in Real Time for Automotive Interfaces: A Multimodal Vision-Based Approach and Evaluations. IEEE Transactions on Intelligent Transportation Systems, pages $1-10,2014$

28. I. Oikonomidis, N. Kyriazis, and A. Argyros. Efficient model-based $3 \mathrm{D}$ tracking of hand articulations using kinect. In $B M V C, 2011$.

29. Y. Pang and H. Ling. Finding the Best from the Second Bests - Inhibiting Subjective Bias in Evaluation of Visual Tracking Algorithms. 2013 IEEE International Conference on Computer Vision, pages 2784-2791, Dec. 2013.

30. A. Pieropan, G. Salvi, K. Pauwels, and H. Kjellstrom. Audio-visual classification and detection of human manipulation actions. In International Conference on Intelligent Robots and Systems, 2014.
31. P. Premaratne, Q. Nguyen, and M. Premaratne. Human computer interaction using hand gestures. Springer, 2010.

32. PrimeSense. Nite2 middleware, 2013. Version 2.2.

33. C. Qian, X. Sun, Y. Wei, X. Tang, and J. Sun. Realtime and robust hand tracking from depth. CVPR 2014.

34. Z. Ren, J. Yuan, and Z. Zhang. Robust hand gesture recognition based on finger-earth mover's distance with a commodity depth camera. In Proceedings of the 19th ACM international conference on Multimedia, pages 1093-1096. ACM, 2011.

35. G. Rogez, M. Khademi, J. S. S. III, J. M. M. Montiel, and D. Ramanan. 3D hand pose detection in egocentric rgb-d images. CDC4CV Workshop, ECCV 2014, 2014.

36. J. Romero, H. Kjellstr, and D. Kragic. Monocular RealTime 3D Articulated Hand Pose Estimation. Humanoid Robots, 2009. Humanoids 2009. 9th IEEE-RAS International Conference on, pages 87-92, 2009.

37. O. Russakovsky, J. Deng, Z. Huang, A. C. Berg, and L. Fei-Fei. Detecting avocados to zucchinis: what have we done, and where are we going? In Computer Vision (ICCV), 2013 IEEE International Conference on, pages 2064-2071. IEEE, 2013.

38. D. Scharstein. A Taxonomy and Evaluation of Dense Two-Frame Stereo. International journal of computer vision, 47(1):7-42, 2002.

39. G. Shakhnarovich, P. Viola, and T. Darrell. Fast pose estimation with parameter-sensitive hashing. In Computer Vision, 2003. Proceedings. Ninth IEEE International Conference on, pages 750-757. IEEE, 2003.

40. J. Shotton, T. Sharp, A. Kipman, A. Fitzgibbon, M. Finocchio, A. Blake, M. Cook, and R. Moore. Realtime human pose recognition in parts from single depth images. Communications of the ACM, 56(1):116-124, 2013.

41. S. Song and J. Xiao. Sliding Shapes for 3D Object Detection in Depth Images. ECCV 2014, pages 634-651, 2014.

42. S. Sridhar, A. Oulasvirta, and C. Theobalt. Interactive Markerless Articulated Hand Motion Tracking Using RGB and Depth Data. Computer Vision (ICCV), 2013 IEEE International Conference on.

43. B. Stenger, A. Thayananthan, P. H. S. Torr, and R. Cipolla. Model-based hand tracking using a hierarchical Bayesian filter. IEEE transactions on pattern analysis and machine intelligence, 28(9):1372-84, Sept. 2006.

44. W. C. Stokoe. Sign language structure: An outline of the visual communication systems of the american deaf. Journal of deaf studies and deaf education, 10(1):3-37, 2005.

45. D. Tang, H. J. Chang, A. Tejani, and T.-K. Kim. Latent regression forest: Structured estimation of $3 \mathrm{D}$ articulated hand posture. Proc. of IEEE Conf. on Computer Vision and Pattern Recognition, 2014.

46. J. Taylor, R. Stebbing, V. Ramakrishna, C. Keskin, J. Shotton, S. Izadi, A. Hertzmann, and A. Fitzgibbon. User-specific hand modeling from monocular depth sequences. In Computer Vision and Pattern Recognition (CVPR), 2014 IEEE Conference on, pages 644-651. IEEE, 2014.

47. J. Tompson, M. Stein, Y. Lecun, and K. Perlin. Real-time continuous pose recovery of human hands using convolutional networks. ACM Transactions on Graphics, 33, August 2014

48. A. Torralba and A. A. Efros. Unbiased look at dataset bias. In Computer Vision and Pattern Recognition (CVPR), 2011 IEEE Conference on, pages 1521-1528. IEEE, 2011. 
49. V. Vezhnevets, V. Sazonov, and A. Andreeva. A survey on pixel-based skin color detection techniques. In Proc. Graphicon, volume 3, pages 85-92. Moscow, Russia, 2003.

50. M. Sarić. Libhand: A library for hand articulation, 2011. Version 0.9.

51. C. Xu and L. Cheng. Efficient Hand Pose Estimation from a Single Depth Image. 2013 IEEE International Conference on Computer Vision, pages 3456-3462, Dec. 2013.

52. Y. Yang and D. Ramanan. Articulated pose estimation with flexible mixtures-of-parts. IEEE Pattern Analysis and Machine Intelligence, 2013.

53. X. Zhu, C. Vondrick, D. Ramanan, and C. Fowlkes. Do we need more training data or better models for object detection?. In $B M V C$, pages 1-11, 2012. 